\title{
NCAP, legislation, and real world requirements require high-pressure dynamics in brake systems
}

Dr. Alexander Brandt, Robert Bosch $\mathrm{GmbH}$

This manuscript is not available according to publishing restriction. Thank you for your understanding. 